\title{
STRESS; THE VULNERABILITY AND ASSOCIATION WITH DRIVING PERFORMANCE
}

\author{
${ }^{1}$ Shamsul, B.M.T., ${ }^{1}$ S. Khairunnisa, ${ }^{1}$ Y.G. Ng and ${ }^{2}$ M.Y. Irwan Syah \\ ${ }^{1}$ Department of Environmental and Occupational Health, \\ Faculty of Medicine and Health Sciences, University Putra Malaysia, 43400 Serdang, Malaysia \\ ${ }^{2}$ Department of Resources Management and Consumer Studies, \\ Faculty of Human Ecology, Universiti Putra Malaysia, 43400 UPM Serdang, Malaysia
}

Received 2013-12-03; Revised 2013-12-13; Accepted 2014-01-11

\begin{abstract}
Several factors may contribute to occurrence of road accidents which are human factors, vehicle factor, road factor and environmental factor. There has been recent evidence of a relationship between road accidents and emotional distress as well as fatigue. Monotonous and complex road environments are the road factor that relates to the internal factor within driver. The overall aim of this study was to compare driving stress, fatigue and driving error between complex and monotonous driving. This experimental study was carried out to measure the stress level, fatigue status and driving performance among hundred male drivers (20-59 years) with driving experience more than one year. Cortisol concentration from respondents' saliva was obtained to measure the stress level due to the driving test. Cortisols were measured using Salimetrics cortisol Enzyme Immunoassay kit (ELISA). Fatigue status was measured by using EEG test to the respondents while conducted the simulator driving test. Driving performance was measured based on the recorded data of Running-of-the Roads (RORI) and Large Speed Variation (LSV) index from the simulator system. This study found that there was a significant difference $(p<0.05)$ between cortisol levels, EEG value and driving errors of monotonous and complex driving. Salivary cortisol level was found higher during monotonous driving compared to complex driving. Theta wave which indicates sleepy and fatigue condition was found higher on monotonous driving compared to other brainwaves which is alpha and beta state. RORI and LSV index was higher recorded during driving in complex road environments. The main implications of this study for road safety shows that monotonous driving had significantly induced driving stress and fatigue while complex driving lead to higher driving errors. Human factors and road factors could possibly put drivers in a higher risk to be involved in road accidents.
\end{abstract}

Keywords: Stress, Driving Performance, Salivary Cortisol, EEG, Simulator System

\section{INTRODUCTION}

Accidents keep increasing throughout the year regardless of the degree of injuries which starts from minor injuries to fatal accident. In urban area, high proportion of accidents involves cyclist $(57 \%)$ or moped riders $(33 \%)$. Moreover, pedestrian constituted $62 \%$ of road fatalities and vehicle group constituted $15 \%$ of road fatalities (ITSDAG, 2013). These high numbered accidents can affect negatively to the country productivity and development since a lot of death occur (Sohadi and Umar, 2005).

There were several factors that contribute to occurrence of road accidents which are human factors, road and vehicles (Behnood and Gohar, 2008). As stated in several studies, road accidents can occurred due to several main factors which are human factor (Stanton and Salmon, 2009), vehicle factor (Yan et al., 2005), road factor and environmental factor (Roge et al., 2004). Sometimes, accidents were not merely Corresponding Author: Shamsul, B.M.T., Department of Environmental and Occupational Health, Faculty of Medicine and Health Sciences, University Putra Malaysia, 43400 Serdang, Malaysia 
occurred by one factor only, they correlates between one factors to another to create events of collision between different vehicles. Variety types of accident had been report to the authorities and it includes those which involved between car driver, lorry and motorcycle rider or to pedestrian and they referred it as Road Traffic Accident (RTA) (Bener, 2005).

From the report of road accident in Malaysia, average accidents that occur keep increasing and need to be put into serious attention. The statistic showed an increasing of $10 \%$ per year which comprises of death, serious injuries, light injuries and loss of property and Malaysia is one of the countries that have high rates of accidents. According to police who issued 133,808 summonses for various traffic offences during the operation, more accidents occurred on federal roads than on highways (SD, 2011).

The main factor of the increase in the road accident is because of human factors such as driving in tired condition that will lead to decrease in concentration and eventually lead to sleeping (Sagberg, 2006). Another mistake that cause by the human factors is because of the driver's attitude. They still drive fast in the area that is dangerous such as at the road corner, around city and also at the highway. Although there are road signages that remind the drivers of the current speed velocity, most of the drivers neglected the road signage. Among all of the causes of accidents, most of them were merely caused by the attitude of the driver.

Stress can have significance impact on driving performance which leads to increase of crashes risk (Beirness, 1993). Matthews (2002) stated that stress may occur when there is a connection between the external demands and internal concerns about personal competency within a specific environment. Thus, stress may relates to specific external situation or events and also the factors reside within an individual (Hill and Boyle, 2007). In this study, stress occurs due to the external factors such as road environment and internal factor within the driver itself.

Fatigue is one of the internal factors that contribute to stress on driver. It is associated with physiological changes in brain wave activity, eye movement, head movement, muscle tone and heart rate. On the onset of fatigue, body temperature, heart rate, blood pressure, respiration rate and adrenalin production are lowered. When fatigued, a person may experience micro-sleeps. Micro-sleeps are brief naps that last for approximately four to five seconds (DGTE, 2009).

Prolonged driving may result symptoms of tiredness, which may be associated with decrements in driving performance (Summala et al., 1999). Fatigue leads to a deterioration of driving performance, diminished steering performance, lesser ability to keep distance to the car in front and increased tendency to mentally withdraw from the driving task (DGTE, 2009). Diminished steering performance and ability to keep distance to the car in front in simulator study can be measured through Running-of-the Roads (RORI) and Large Speed Variation (LSV) index which also represents the driving errors.

In Malaysia, research regarding stress and driving performance was not as much as those in other countries. Therefore this approach is one of the methods to understand the factors and mechanism of accidents occurrence in order to reduce the incident. The use of saliva instead of other samples (blood, urine) has the advantage in term of the stress development. Moreover, using saliva as a biomarker will not affect stress level compared to other parameter.

\section{MATERIALS AND METHODS}

\subsection{Driving Simulator System}

A realistic custom made driving simulator system with modified vehicle instruments and controls such as steering wheel, foot pedals and gear lever was shown in Fig. 1. A driving simulator can measure performance accurately and efficiently (De Winter et al., 2009). A video screen was made as the "in-car" view and projected with a 32 inch LCD screen television located about 1 meter from the driver. The display unit on screen reproduced town area, trunk road and motorway scenery with computer-generated pictures simulating daytime and night time driving condition. A total of 5 laps driving journey (each lap $=50 \mathrm{~km}$ ) was simulated with different sceneries. All the simulated roadways were in two lanes. Drivers were allowed to overtake the in front vehicles. The speed limit legalized in town area, trunk road and motorway was 50,60 and $90 \mathrm{~km} \mathrm{~h}^{-1}$ respectively.

\subsubsection{Driving Performance Measures}

Driving errors were measured in terms of number of Running-of-the roads (RORI) index and Large Speed Variation (LSV). The method of calculating driving errors was adapted by Campagne et al. (2004). RORI index was measured for each second by the amplitude of the exit. It consisted of crossing over to the Emergency Lane (EL) or the continuous white line on the left-hand hedge of the passing lane. When the vehicle stays in the traffic lanes, the score was 0 . Otherwise, it equals the distance between left front wheel and the EL for a RORI on the left-hand side and the distance between the right edge line for a 
RORI in the Central Reservation (CR). The penalty was calculated for $1 \mathrm{~min}$ and corresponds to the square root of the sum of the RORI amplitudes squared for each second:

Running-off-the-road index: Penalty/min=( amplitude2)

For Large Speed Variation (LSV), a subject was penalized if an absolute difference between the vehicle speed and the highest speed authorized in the segment of road considered was calculated for each second. If the absolute difference is more than $20 \mathrm{~km} \mathrm{~h}^{-1}$, the subject will get a penalty (per second) proportional to the number of kilometers per hour above the speed authorized. The sum of penalty is made for each minute on the whole experiment (Campagne et al., 2004).

$$
\text { If } \Delta \text { speed }>20 \text {, then penalty }(\text { per second })=(\Delta \text { speed } / 10)-1
$$

\subsection{Electroencephalographic (EEG)}

The Electroencephalogram (EEG) is a test that measures and records the electrical activity of brain by attached the special sensor to head (scalp) and record the brain's spontaneous electrical activity. It is one of the most valid indexes to measure alertness in the driver (Lin et al., 2005). The Electroencephalograph (EEG) measures brainwaves of different frequencies within the brain. This activity appears on the screen of the EEG machine as waveforms of varying frequency and amplitude measured in voltage (Blume and Kaibara, 1999). The EEG is traditionally analyzed in terms of temporal waveforms at certain channels, looking at power of rhythms in the spontaneous EEG, at amplitude and latency of the peaks and troughs in Event Related Potentials (ERPs), or at particular grapho-elements in pathological or sleep stages. EEG in this study was used to measure the fatigue condition of respondents during driving through the EEG waveforms frequency (alpha, beta and theta). Figure 2 shows EEG was used during driving on simulator system by attached the sensors on respondent's head.

\subsubsection{EEG Waveforms}

The electrical activity of the brain is classified according to rhythms. The EEG activity in this study was defined in terms of three frequency bands including theta $(6-8 \mathrm{~Hz})$, alpha $(8-12 \mathrm{~Hz})$ and beta (13$30 \mathrm{~Hz}$ ) (Teplan, 2002; Schier, 2000). This brainwaves pattern associated with all sorts of emotional and neurological conditions. Thus, this EEG was used to interpret the condition of brainwaves while driving using the simulator system which also represents the same condition of brain during driving in real situation.

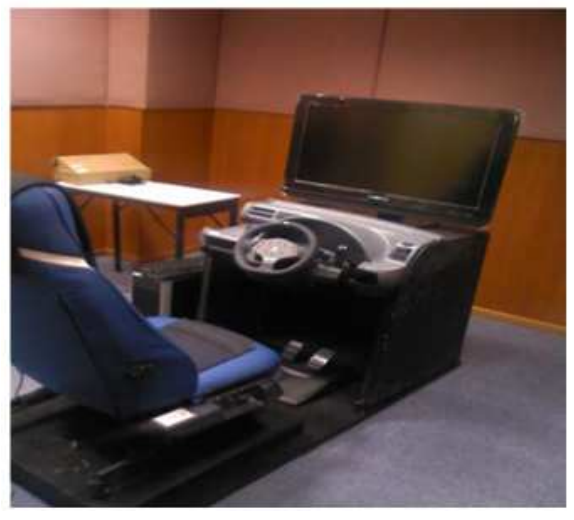

Fig. 1. Driving simulator system

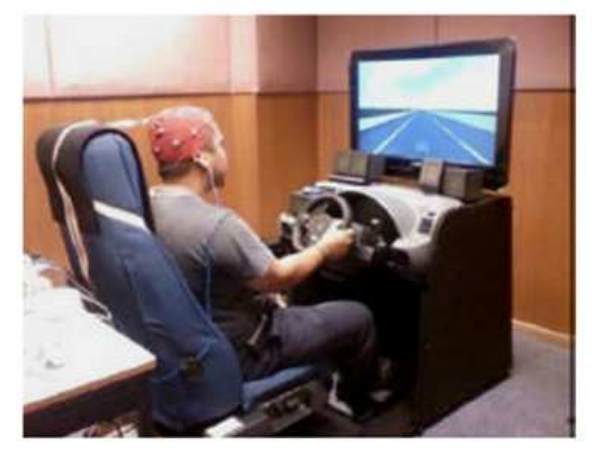

Fig. 2. Electroencephalogram (EEG) test

\subsection{Salivary Cortisol Analysis}

Salivary cortisol may provide a better measure than serum cortisol of the stress response as it more accurately measures the amount of unbound cortisol compared to serum measures (McGuigan et al., 2004). Saliva samples were collected using saliva collecting device after the respondent undergoes the driving performance test by using the simulator. Those samples was then stored in temperature below $-20^{\circ} \mathrm{C}$ within $30 \mathrm{~min}$ after collection in order to avoid bacterial growth. The analysis was performed using Salimetrics cortisol kit. The Salimetrics cortisol kit is a competitive immunoassay specifically designed and validated for the quantitative measurement of salivary cortisol. The desired number of saliva in the frame holder was secured during analysis. This kit is a solid phase Enzyme-Linked Immunosorbent Assay (ELISA), based on the principle of competitive binding (Salimetrics, 2011). 


\subsubsection{Stress Level Measures}

The saliva samples obtained from the respondents towards the end of the driving performance test were to measure the concentration of their salivary cortisol which indicates the stress level during the driving test. Increasing levels of cortisol act as negative feedback signal. Free unbound cortisols tend to increase further before to another 15 to $20 \mathrm{~min}$ before beginning to decrease (King and Hegadoren, 2002). It is used to measure the stress level through the concentration of salivary cortisol among respondents. The higher concentration of salivary cortisol indicates higher stress level felt during the driving test among the respondents.

\subsection{Statistical Analysis}

Data were entered and analysed using the Statistical Packages for Social Sciences (SPSS) version 19. Cortisol level, EEG value and driving error index for both monotonous and complex driving were analysed using paired t-test. The association of the monotonous and complex driving variables was considered significant at a value of $\mathrm{p}<0.05$.

\section{RESULTS}

\subsection{Socio-Demographic Data of Respondents}

Hundreds male drivers, aged between 20-59 years old and had driving experience more than one year, were recruited in this study. Respondents were categorized into group of years of driving, age, highest education and miles of driving (per week). The highest years of driving group in this study were among group of 25 to 30 years of driving $(n=25,25 \%)$. The lowest was among 1 to 5 years of driving group $(n=3,3 \%)$. The distribution of ages among respondents are equally distributed which are $25 \%(n=25)$. Most of the respondents have degree as their highest education back-ground. Demographic characteristics of respondents are presented in Table $\mathbf{1 .}$

\subsection{Cortisol Level Value Between Complex and Monotonous Driving}

Table 2 shows that there was significant difference $(p<0.05)$ found on cortisol level between monotonous and complex driving. Respondent's level of cortisol when driving in monotonous road environments was higher with mean and standard deviation of $4.169 \pm 1.308$ compared to complex road environments which is $3.865 \pm 1.288$.
Table 1. Socio-demographic characteristic of respondent

\begin{tabular}{ll}
\hline Variable & Frequency $(\%)$ \\
\hline Year of driving & $3(3 \%)$ \\
1-5 years & $13(13 \%)$ \\
5-10 years & $21(21 \%)$ \\
10-15 years & $17(17 \%)$ \\
15-20 years & $21(21 \%)$ \\
$20-25$ years & $25(25 \%)$ \\
$25-30$ years & \\
Age & $25(25 \%)$ \\
$20-29$ years old & $25(25 \%)$ \\
$30-39$ years old & $25(25 \%)$ \\
$40-49$ years old & $25(25 \%)$ \\
$50-59$ years old & \\
Highest education & $43(43 \%)$ \\
SPM & $54(54 \%)$ \\
Degree & $3(3 \%)$ \\
Master &
\end{tabular}

Table 2. Comparison of cortisol level between monotonous and complex driving

\begin{tabular}{lccll}
\hline Variable & Mean & $\begin{array}{l}\text { Standard } \\
\text { deviation }\end{array}$ & $\begin{array}{l}\mathrm{t}- \\
\text { value }\end{array}$ & $\begin{array}{l}\mathrm{p}- \\
\text { value }\end{array}$ \\
\hline $\begin{array}{l}\text { Cortisol } \\
\text { Monotonous }\end{array}$ & 4.169 & 1.308 & 2.23 & $0.028^{*}$ \\
Complex & 3.865 & 1.288 & & \\
\hline
\end{tabular}

*Significant at $\mathrm{p}$ value $<0.05$

\subsection{EEG Value Between Complex and Monotonous Driving}

Table 3 shows that there was significant difference $(p<0.05)$ of EEG values between monotonous and complex driving. Theta waves shows highest mean observed compared to alpha and beta waves which were $0.221 \pm 0.047$ for monotonous and $0.204 \pm 0.044$ for complex road environments. Alpha waves showed the lowest mean value with $0.052 \pm 0.042$ for monotonous driving and 0.048 \pm 0.044 for complex driving.

\subsection{RORI and LSV Value Between Complex and Monotonous Driving}

Table 4 shows that there were significant difference $(p<0.05)$ found between driving error (RORI and LSV) of monotonous driving and complex driving. The mean of RORI was higher observed when driving in complex road environments $(62.41 \pm 16.60)$ compared to $56.41 \pm 11.10$ when driving in monotonous road environments. The same goes for the LSV, driving in complex road also shows higher mean $(128.81 \pm 23.52)$ compared to $120.02 \pm 18.14$ when driving in monotonous road environments. 
Table 3. Comparison of EEG values between monotonous and complex driving

\begin{tabular}{lclll}
\hline Variable & Mean & $\begin{array}{l}\text { Standard } \\
\text { deviation }\end{array}$ & $\begin{array}{l}\mathrm{t}- \\
\text { Value }\end{array}$ & $\begin{array}{l}\mathrm{p}- \\
\text { Value }\end{array}$ \\
\hline EEG & & & & \\
Alpha (monotonous) & 0.0524 & 0.04283 & 3.822 & $0.001^{*}$ \\
Alpha (complex) & 0.0483 & 0.04476 & & \\
Beta (monotonous) & 0.1861 & 0.0605 & -5.958 & $0.001^{*}$ \\
Beta (complex) & 0.2003 & 0.04935 & & \\
Theta (monotonous) & 0.2214 & 0.04776 & 6.835 & $0.001^{*}$ \\
Theta (complex) & 0.2044 & 0.04473 & & \\
\hline
\end{tabular}

*Significant at $\mathrm{p}$ value $<0.05$

Table 4. Comparison of driving errors between monotonous and complex driving

\begin{tabular}{lclll}
\hline Variable & Mean & $\begin{array}{l}\text { Standard } \\
\text { deviation }\end{array}$ & $\begin{array}{l}\mathrm{t}- \\
\text { value }\end{array}$ & $\begin{array}{l}\text { p- } \\
\text { value }\end{array}$ \\
\hline RORI (monotonous) & 56.406 & 11.909 & -2.966 & $0.040^{*}$ \\
RORI (complex) & 62.411 & 16.604 & & \\
LSV (monotonous) & 120.024 & 18.143 & -2.987 & $0.040^{*}$ \\
LSV (complex) & 128.807 & 23.521 & & \\
\hline
\end{tabular}

*Significant at $\mathrm{p}$ value $<0.05$

\section{DISCUSSION}

Cortisol level when driving in monotonous road environments was highly observed. This shows that driving in monotonous road environments lead to emotional distress. Cortisol level in saliva was an indicator to measure stress level of a person. This result proved that driving in monotonous road environments can be one of the indirect sources of stress. Emotional distress in this situation may come from the results of fatigue due to extreme focus needed during driving in such condition. Fatigue may lead to stress as the driver's was struggled to maintain concentration is itself threatening to safety (Desmond and Matthews, 2009).

The EEG values were classified according to rhythms, which are defined in terms of frequency bands including alpha, beta and theta. Theta waves presence was to indicate relaxed, meditative and creative state in healthy individual. As from the results, it showed that theta waves were higher in monotonous driving. Theta waves are a condition where a person is in between conscious and subconscious state, which are when we are most relaxed but not asleep. It is most likely the onset of sleep. This relaxed but aware quality is exactly why so many people claim to have their best ideas while in the shower, driving, or drifting off to sleep (Hines, 2012). The repetitive or calming aspect of driving in monotonous road environments puts people in this condition to be more in theta state.
Based on the results, theta waves was slightly higher during monotonous driving compared to complex driving. Previous study stated that characteristics of road geometry and road side environment which is monotonous road environment can have an impact on driving performance by affecting arousal, decrease alertness and vigilance (Thiffault and Bergeron, 2003). Also, driving on a relatively long and monotonous driving environment also has a clear negative effect on driver valid peripheral visual field (Roge et al., 2003).

Driving error (RORI and LSV) was found higher observed during driving in complex road environments. This result showed that complex road environments leads to errors occurred while driving. In line with previous study, by Seen et al. (2010) found that higher driving errors was occurred on complex road such as town or trunk road area compared with highway which is monotonous road environments. The pattern of complex road environments itself may cause the driver to be less alert especially when deal with turns and junctions on road.

\section{CONCLUSION}

From the findings of this study, there was significant difference $(p<0.05)$ found between cortisol levels, EEG value and driving errors of monotonous and complex driving. Respondents who drive in monotonous road environments was found emotionally distress based on the salivary cortisol level obtained among them. Stress happen in this situation was due to the fatigue condition. Fatigue while struggling to maintain the concentration on driving will cause stress to the driver itself. This was showed through the results of EEG value of respondents. Theta waves was found higher on complex and monotonous driving compared to other brainwaves which is alpha and beta state. Theta state is a condition where we are in a stage of between conscious and subconscious. It occurs most often in sleep. This shows that prolonged driving especially in monotonous road environments may cause fatigue and sleepy. EEG tests during driving is a good measurement for this study as it recorded possible correlation between physical and mental state of driver, driving behaviour and scenario conditions such as the road conditions (Rimini-Doering et al., 2001).

In this study, driving errors was measured using the RORI and LSV index from the driving simulator recorded data. Results show that both RORI and LSV were higher during complex driving. Complex road environments significantly cause the driver to be less control and repeatedly crossing over the line on side of the roads. 
Stress and fatigue during driving was one of the internal factors lead to road accidents. This study found that monotonous road environments cause stress level to increase as the driver encounter fatigue during driving. Thiffault and Bergeron (2003) supported that driver steering wheel movement is greater and occurs more often in a monotonous driving situation, showing that the fatigue effect and effect on driver vigilance caused by a monotonous road situation is relatively large. However, RORI and LSV index was higher on complex driving due to the patterns of road design and lack of alertness of the driver itself to control the speed of driving on turns and junctions road. Despite the road conditions itself, human factors are still being a part of road accidents causes.

A possible limitation of this research is the use of a simulator to study driving performance of varying road environments. Some argue that laboratory based study is often criticised for a lack of realism and unrepresentative results of field settings (Tredoux and Pooley, 2001). However, the use of driving simulator in this study controlled the extraneous factors of field settings such as distractions from other vehicle or driver's behaviours on road which is not the main focus of this study. This study could be as a preliminary research in a simulated setting prior to test the findings on real context of driving. Thus, this study suggested future research on driving performance in field settings. Continued research in this study also will be strengthened by using the Driver Stress Inventory (DSI) to measure an individual's vulnerability to stress reactions during driving.

\section{ACKNOWLEDGEMENT}

This study was financially funded by the Research University Grant Scheme, Initiative 6 (Grant No.: 9318990), of the Universiti Putra Malaysia.

\section{REFERENCES}

Behnood and A.R., PakGohar, 2008. The preliminary plan for budgeting the costs of social training of traffic. M.R. the organization of transportation and traffic management, 2008. Proceedings of the 8th Conference on Transportation Engineering and Traffic in Iran, (TETI' 08).

Beirness, D.J., 1993. Do we really drive as we live? The role of personality factors in road crashes. Alcohol, Drugs Driv., 9: 129-143.

Bener, A., 2005. The neglected epidemic: Road traffic accidents in a developing country, State of Qatar. Int. J. Injury Control Safety Promot., 12: 45-47. DOI: $10.1080 / 1745730051233142225$
Blume, W.T. and M. Kaibara, 1999. Atlas of Pediatric Electroencepha-Lography. 2nd Edn., LippincottRaven, Philadelphia, ISBN-10: 0781714346, pp: 391.

Campagne, A., T. Pebayle and A. Muzet, 2004. Correlation between driving errors and vigilance level: Influence of the driver's age. Physiol. Behav., 80: 515-524. DOI: 10.1016/j.physbeh.2003.10.004

De Winter, J.C.F., S. De Groot, M. Mulder, P.A. Wieringa and J. Dankelman et al., 2009. Relationships between driving simulator performance and driving test results. Ergonomics, 52: 137-153. DOI: 10.1080/00140130802277521

Desmond, P.A. and G. Matthews, 2009. Individual differences in stress and fatigue in two field studies of driving. Trans. Res. Part F: Traffic Psychol. Behav., 12: 265-276. DOI: 10.1016/j.trf.2008.12.006

DGTE, 2009. Fatigue. EU Commission, DirectorateGeneral Transport and Energy.

Hill, J.D. and L.N. Boyle, 2007. Driver stress as influenced by driving maneuvers and roadway conditions. Trans. Res. Part F: Traffic Psychol. Behav., 10: 177-186. DOI: 10.1016/j.trf.2006.09.002

Hines, B., 2012. Tapping into creativity with theta brain wave cycles. Marketing with Creativity.

ITSDAG, 2013. IRTAD: An international expert network and database on road safety Data. Int. Traffic Safety Data Anal. Group.

King, S. L. and K.M. Hegadoren, 2002. Stress hormones: How do they measure up.? Biol. Res. Nurs., 4: 92103. DOI: $10.1177 / 1099800402238334$

Lin, C.T., Wu, R.C., Jung, T.P., Liang, S.F., Huang, T.Y., 2005. Estimating driving performance based on EEG spectrum analysis. EURASIP J. Applied Signal Process., 19: 3165-3174.

Matthews, G., 2002. Towards a transactional ergonomics for driver stress and fatigue. Theoretical Issues Ergonomics Sci., 3: 195-211. DOI: 10.1080/14639220210124120

McGuigan, M.R., A.D. Egan and C. Foster, 2004. Salivary cortisol responses and perceived exertion during high intensity and low intensity bouts of resistance exercise. J. Sports Sci. Med., 3: 8-15.

Rimini-Doering, M., D. Manstetten, T. Altmueller, U. Ladstaetter and M. Mahler, 2001. Monitoring driver drowsiness and stress in a driving simulator. Proceedings of the International Driving Symposium on Human Factors in Driver Assessment, Training and Vehicle Design, (TVD' 01), Iowa University, Iowa City, pp: 58-63. 
Roge, J., T. Pebayle, E. Lambilliotte, F. Spitzenstetter and D. Giselbrecht et al., 2004. Influence of age, speed and duration of monotonous driving task in traffic on the driver's useful visual field. Vis. Res., 44: 2737-2744. DOI: 10.1016/j.visres.2004.05.026

Roge, J., T. Pebayle, S.E. Hannachi and A. Muzet, 2003. Effect of sleep deprivation and driving duration on the useful visual field in younger and older subjects during simulator driving. Vis. Res., 43: 1465-1472. DOI: 10.1016/S0042-6989(03)00143-3

Sagberg, F., 2006. Driver health and crash involvement: A case-control study. Accident Anal. Prevent., 38: 28-34. DOI:10.1016/j.aap.2005.06.018

Salimetrics, L., 2011. High sensitivity salivary cortisol enzyme immunoassay kit.

Schier, M.A., 2000. Changes in EEG alpha power during simulated driving: A demonstration. Int. J. Psychophysiol., 37: 155-162. DOI: 10.1016/S01678760(00)00079-9

SD, 2011. 289 deaths the worst on Ops Sikap record. The Sun Daily.

Seen, K.S., S.B.M. Tamrin and G.Y. Meng, 2010. Driving fatigue and performance among occupational drivers in simulated prolonged driving. Global J. Health Sci., 2: 167-167.
Sohadi, R. and R. Umar, 2005. Updates of road safety status in Malaysia. IATSS Res., 29: 78-80.

Stanton, N.A. and P.M. Salmon, 2009. Human error taxonomies applied to driving: A generic driver error taxonomy and its implications for intelligent transport systems. Safety Sci., 47: 227-237. DOI: 10.1016/j.ssci.2008.03.006

Summala, H., H. Hakkanen, T. Mikkola and J. Sinkkonen, 1999. Task effects on fatigue symptoms in over-night driving. Ergonomics, 42: 798-806. DOI: $10.1080 / 001401399185298$

Teplan, M., 2002. Fundamentals of EEG measurement. Measurement Sci. Rev., 2: 1-11.

Thiffault, P. and J. Bergeron, 2003. Monotony of road environment and driver fatigue: A simulator study. Accident Anal. Prevent., 35: 381-391. PMID: 12643955

Tredoux, C. and S. Pooley, 2001. Polygraph based testing of deception and truthfulness: An evaluation and commentary. Indus. LJ, 22: 819-819.

Yan, X., E. Radwan and M. Abdel-Aty, 2005. Characteristics of rear-end accidents at signalized intersections using multiple logistic regression model. Accident Anal. Prevent., 37: 983-995. DOI: 10.1016/j.aap.2005.05.001 